\title{
Explicit Parametrizations of the Configuration Spaces of Anthropomorphic Multi-Linkage Systems
}

\author{
Li Han and Lee Rudolph \\ Department of Mathematics and Computer Science \\ Clark University \\ Worcester, MA 01610 \\ Email: [lhan, lrudolph]@clarku.edu
}

\begin{abstract}
Multi-fingered manipulation systems are important in the study of robotics. These are also challenging systems, in part because of the loop closure constraints required of several (virtual) loops each formed by two fingers and the grasped object. Most existing work describes system configurations using joint parameters, in which loop closure constraints are expressed by highly nonlinear equations. Such a formulation amounts to an implicit parametrization of the configuration space (CSpace) as a lower-dimensional semi-algebraic subset embedded in a higher-dimensional ambient joint parameter space. The non-zero difference between the two dimensions is the codimension of CSpace as seen in the given parametrization.

In this paper, we point out that, quite generally, parametrizations leading to lower codimensional configuration spaces provide multi-faceted advantages over those producing higher codimensions. For two example manipulation system-a 3-fingered hand and a planar star-manipulator with any number of fingerswe present explicit parameterizations, which are effectively of codimension 0 . We base these parametrizations on our recently developed construction trees of simplices (such as triangles and tetrahedra) for multi-object systems; such a tree gives simplexbased parametrers on CSpace, in which loop closure constraints become simplex formation constraints (such as triangle inequalities and Cayley-Menger determinant constraints). Both example systems are very difficult to deal with using joint angle parameters. Our results here further demonstrate the generality and effectiveness of the simplex-based approach.
\end{abstract}

\section{OVERVIEW}

Multi-linkage robotic systems, such as robotic hands and legs, have great potential in diverse areas; they have been intensively researched for several decades(see e.g. books [4], [5], [18]-[20], [22], the recent white paper [7], and references therein). These challenging systems are subject to complicated constraints, among them loop closure constraints arising from (virtual) loops formed by multiple limbs all touching one objects. Conventional parameters for these systems are joint parameters, in which loop closure constraints are formulated as nonlinear equations. In such a formulation, the configuration space (CSpace) of a system is a semi-algebraic or semianalytic set, generically a smooth manifold, embedded in its ambient joint space (for an example, see the study of singleloop systems with spherical-type joints [21], [28]). Such an embedding gives an implicit parametrization of CSpace: by the Implicit Function Theorem, any given configuration point has a CSpace neighborhood on which some subset of the ambient space parameters are coordinates for a local chart, but to effectively find an atlas of such charts covering CSpace, and to compute properties of these charts like their geometries and mutual intersections, is not easy. The lack of methods to access such local information becomes a huge hurdle in the study of the global structure of CSpace.

The codimension of a subset in an ambient space is the difference between its dimension and that of the ambient space; thus, in 3-dimensional Euclidean space, a curve has codimension 2, a surface has codimension 1, and a nonempty open subset (such as the set of points enclosed by a sphere) has codimension 0 . By an explicit parametrization of an open subset $U$ of CSpace, we mean one whose chart has codimension 0: that is, $U$ is (explicitly) identified with an open set in Euclidean space of the same dimension as CSpace. An explicit atlas for CSpace is one in which all charts have codimension 0; with an explicit atlas in hand, we do not have to study CSpace as a lower-dimensional subspace of a higherdimensional space. As an example, consider the unit circle $C$ in the $(x, y)$-plane. With $x$ and $y$ coordinates as implicit parameters for $C$, the smallest atlas comprises four charts (semicircles). In contrast, the polar angle $\theta$ (on any interval of length less than $2 \pi$ ) is an explicit parameter, and yields an explicit atlas comprising just 2 charts. More generally, any simple closed curve $\Gamma$ has a 2 -chart explicit atlas coordinatized by arc length, but for any $n>2$ there exists $\Gamma$ for which any atlas with implicit parameters $x$ and $y$ needs $n$ charts.

As briefly discussed in Section II, there are many advantages to the development and use, when possible, of parameters for CSpace that reduce codimension (explicit parameters being optimal). There are also advantages - which can sometimes conflict with codimension reduction-to parameters in which constraint formulations are particularly simple.

In this paper, we present explicit parametrizations for two example anthropomorphic linkage systems. (1) The 3-fingered manipulation system in Fig. 1 has 14 degrees of freedom, dropping to 11 when it grasps an object with all 3 fingers at fixed contacts. In joint angle parameters, the 11-dimensional configuration space of the grasping hand has codimension 3 in a 14-dimensional torus, and the 3 loop closure constraint equations are complicated trigonometric expressions. In joint Cartesian coordinates, the same 11-dimensional CSpace is realized as a codimension-22 submanifold; although the defining equations are quadratic polynomials, the loop closure 


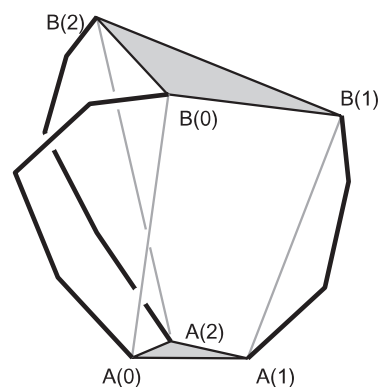

Fig. 1. A model grasp system with 3 fingers; each has a spherical base joint, and intermediate revolute joints with parallel axes.

constraints make the implicit parameters highly coupled and difficult to solve. By contrast, our simplex-based parameters for this CSpace are explicit, and the loop closure constraints are straightforward simplex-formation constraints (triangle and Cayley-Menger determinant inequalities). This parametrization provides a solid foundation for the study of system configurations, and can be used to solve the global structure of CSpace and develop efficient manipulation planning algorithms. We discuss it in section III. (2) Recent papers [17], [25] have studied motion planning for star-manipulators, a class of planar closed-chain manipulators. In section IV we present explicit parametrizations for all star-manipulators.

\section{EXPlicit PARAmeters: Motivations, Prior WORK}

In view of complex closure constraints in the joint parameters, recent results on the explicit parametrization and solved structures of one type of systems with loops become even more remarkable. In short, for a planar single-loop linkage with $n$ revolute joints (equivalently, a planar $n$-sided polygon), fixed link lengths, and having 3 "long links" (a technical condition defined in [21], [28]), it is proved [14], [21], [28] that one set of explicit configuration parameters consists of joint angles of $n-3$ "short links", and the configuration space under such a parametrization comprises two disjoint $(n-3)$-dimensional tori (the two components correspond to two possible orientations of the 3 long links - "elbow up" and "elbow down"). The existence of two components in CSpace reflects the fact that, while a loop without 3 long links can be reoriented (if link crossings are allowed), one with 3 long links cannot be. We will call this property un-reorientability.

The explicit parametrization and solved structures just mentioned greatly facilitate computations for un-reorientable planar loops. For example, any values for the $n-3$ parameter angles will correspond to valid loop configurations. Also, a torus can be cut open into a cube, which, being convex, allows trivial path planning (any two points can be connected by a line segment); it follows that given two identically oriented configurations of an un-reorientable planar loop, it is trivial to find a path between them (ignoring collision or any other constraints), by computing and "linearly" interpolating parameter angle values. (Of course, whereas two points in a truly convex set are joined by a unique line segment, in a torus there are many such segments; all but one cross one or more "cuts", and all are equally trivial to compute.)
A linear path of a un-reorientable planar loop satisfies the loop closure constraints and stays on one of the two CSpace tori. But it may involve link penetrations. Extensive research by the motion planning community has made it clear that collision-avoidance is very difficult to deal with, and that it is very hard to find analytic descriptions of the subset CFree of valid configurations, and its complement CObstacle, for general obstacles. Recent successes of randomized path planners (see books [3], [15], [16] and references therein) suggest that sampling-based approaches may be an important framework in which to integrate efficient sampling methods while also dealing with such difficult factors in configuration space and path planning problems as high dimensionality and complicated linkage constraints. Sampling-based approaches can handle complicated constraints (by checking a sample point against the constraints, rather than trying to find all solutions to the constraints) and can be used with implicit surfaces in ambient spaces, but sampling efficiency can be improved by explicit parametrization (or, more generally, low codimension). This is due to two facts: for a purely random sampling scheme, the statistical success rate for generating valid samples in a subset equals the volume of the valid subset divided by the volume of the entire sample space; and, given an acceptable error $\varepsilon>0$, the volume of an $\varepsilon$-neighborhood of a submanifold of codimension $k>0$ tends to 0 like $\varepsilon^{k}$.

Thus, for example, consider the problem of generating one collision-free loop configuration, that is, a point in CFree. In general CFree is open in CSpace, and thus has the same dimension as CSpace. Consequently, if we have an explicit parametrization of CSpace, we can take any explicit chart as a sample space, and our success rate for generating a collision-free loop configuration in that chart will be the volume of the part CFree in that chart divided by the volume of the chart. If this rate is 0 for a particular chart, it means that the chart actually misses CFree, and there may even be an understandable structural reason why (see [13] for examples); if this ratio is positive, valid configurations should be found with positive probability. In contrast, if we only have parameters for the ambient space (e.g., the joint parameter space) and an implicit parametrization for the configurations (as defined by the closure constraints in joint parameters), then the sampling space is the ambient space, the configuration space has codimension $d \geq 1$, and as stated above this means that the probability of finding any loop configurationlet alone a collision-free configuration-by random sampling in the ambient space approaches 0 like the $d$ th power of the acceptable error. By analogy with the well-known "curse of dimensionality", that the number of samples needed to adequately cover a sample space increases exponentially with the dimension of the space, we propose the name "curse of codimensionality" for the situation just described: that, other things being equal, the higher the codimension of a target space in its ambient space, the more challenging it is to sample it efficiently (the challenge increasing with the codimension).

Given that parameters corresponding to lower-codimension parametrizations tend to provide multifaceted advantages for 
configuration related problems (aiding analytical study of global CSpace structure, facilitating sampling, etc.), finding such parameter systems is a problem of considerable interest. Systems without loops, like rigid bodies and open chains, have well-known and widely used explicit parametrizations, e.g., various 6 -DOF parameters for a $3 D$ object or joint parameters for an open chain. However, to the best of our knowledge, rather few systems involving loops have been given explicit parametrizations in prior work: (a) single-loop planar linkages with revolute joints and 3 long links [14], [21], [28]; (b) trilaterable manipulators [24]; (c) flag manipulators [1], [27].

Loosely speaking, a system is "trilaterable" in the sense of [24] if it can be decomposed into tetrahedra in such a way that all unknown edge-lengths of the tetrahedra can be systematically computed from known edge-lengths using distance constraints (triangle inequalities and Cayley-Menger constraints; see below); flag manipulators generalize trilaterable systems. Note that the solutions in [1], [24], [27] assume systems already given in trilaterated form, with kinematic structures explicitly including all distance parameters needed to determine system configurations (e.g., lengths of the legs between base and platform of a parallel manipulator).

Our Work. We have recently developed simplex-based approaches for multibody systems that allow construction trees of simplices (such as triangles and tetrahedra). The main represenative systems used in our published papers, which describe their explicit paramatrizations and some other issues, are these: (a) single-loop planar linkages with revolute joints [8], [11][13]; (b) single-loop spatial linkages with spherical joints [8], [9]; (c) single-vertex rigid origami folds [10]. Along with some simple examples, we have pointed out in these papers that the described approaches are directly applicable to multi-loop systems having simplicial construction trees. (Our approaches can also easily accomodate link length constraints, which can be used to model prismatic joints along the links, link length tolerance and uncertainty, and so on.)

In this paper we present explicit parametrizations for examples of multi-loop linkage systems with anthropomorphic structures, which would have been very hard to study using the conventional joint parameters. Our first example system comprises 3 fingers with spherical base joints, revolute intermediate joints, and tips that we model (when the hand is grasping an object) as spherical joints; one motivational problem for this system is gaited 3-fingered manipulation (see Fig. 2). Our second example system is a general starmanipulator (as in Fig. 6). The example systems, and their
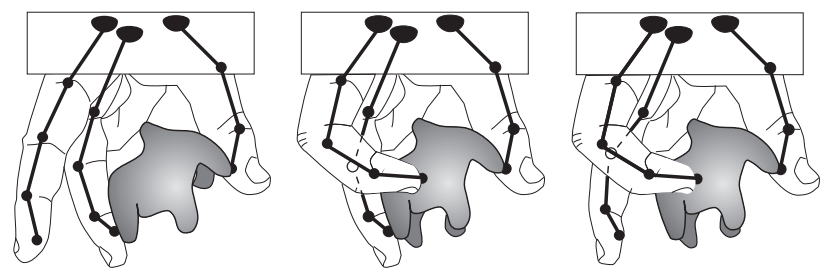

Fig. 2. Manipulation: gaiting between two 2 -finger grasps via a 3-finger grasp. simplicial construction trees, are substantially different from those in our earlier. Here we will focus on their explicit simplex-based parameters. Our results further demonstrate the versatility and effectiveness of the simplex-based approach.

Remarks. (1) We use the term "grasp" purely kinematically, and thus ignore such important practical issues as force-closure (see [23]). (2) Multi-limb systems using finger gaiting and legged locomotion have been studied before, as kinematic stratified systems [6], [29]; the main focus of that work was control issues, and those papers describe configuration spaces as coarse-grained stratified spaces, with each grasp mode (having the object grasped by one particular subset of fingers) corresponding to one conceptual, implicit stratum, embedded (as usual) in the ambient parameter space. (3) The "reachable distances" approach to motion planning for closed chains given in [26] is closely related to our work on closed chains, and it would be interesting to see how such an approach might apply to the example systems in this paper.

\section{EXAMPLE System I: An ANTHROPOMORPHIC HAND}

Our example 3 -fingered hand system as drawn in Fig. 1 is an idealized model of the human hand shown in Fig. 2, where we model each finger link by the line segment between its two end points and do not consider the rotational movement about the links. Further, we consider the fixed base joints $B_{i}$ ( $i=0,1,2$, modulo 3 ) to be spherical, and all other link joints to be revolute; further, on each finger, the axes of all the revolute joints are parallel to each other and perpendicular to the finger plane. Of the three fingers, one (thumb) has 3 links, and the other two have 4 links each, so in total the system has 14 degrees of freedom. As shown in Fig. 3, we use the following 14 inter-joint diagonals to define a tree of simplices (triangles and tetrahedra in this case) for such a system.

- 3 diagonals between the fingertips, $[A(0) A(1)]$, $[A(1) A(2)],[A(2) A(0)]$

- 3 diagonals between the finger tips and bases, $[A(i) B(i)]$, $i=0, \ldots, 2$

- 3 diagonals for decomposing the (virtual) parallel 3platform formed by $\{A(i), B(i), i=0, \ldots, 2\}$ into three tetrahedra (as shown in the top row of Fig. 3, $[A(1) B(0)]$, $[A(2) B(0)],[A(20 B(1)]$

- 5 diagonals for decomposing each virtual finger loop (closed by the base-to-tip diagonal) into a tree of triangle, such as those shown at the bottom row of Fig. 3 for trees of triangles anchored at the tip joints - a planar $n$-gon has $n-3$ diagonals for a triangle tree (for more details, see [8], [11]); in total, the three fingers have 5 such diagonals, one for the thumb, and two each for the other two fingers.

\section{A. Simplex-Based Parameters}

The simplices in Fig. 3 form the major part (with a subtlety and some additional simplices to be discussed in subsection III-C) of a simplicial construction tree of this system, that is, a tree of simplices satisfying the following three conditions. (1) Each link in the linkage system is an edge of at least one simplex in the tree. (2) The set of joints of the 
linkage system equals the set of all vertices of all simplices in the tree. (3) The configurations of the linkage system can be constructed from the shapes of the simplices and relative configurations of simplices adjacent in the tree.

These three properties enable us to construct configurations using the simplex assembly process. To see this, note that placing a simplex in an ambient space is equivalent to determining the coordinates of its vertices. Now, given the shapes and relative configurations of the simplices, we construct the corresponding configuration of the system recursively as follows. (I) Fix the initial tetrahedron (along with the fixed base joints) in place. (II) While there is an edge in the tree such that one simplex has already been placed in space but the other simplex has not yet been placed, then use the relative configuration between the two to place the other one. When this simplex assembly process terminates, the configuration has been constructed.

Our simplex based parameters for the system comprise

- shape parameters (for the shapes of the simplices): lengths of the diagonals

- orientation parameters (for the relative configurations of adjacent simplices).

There are generally two types of orientation parameterseither a continuous angle (a dihedral angle), e.g., for two spatial simplices sharing one edge, or a discrete (essentially binary-valued) orientation sign, e.g., for two planar triangles sharing an edge or two spatial tetrahedra sharing a face.

These binary orientations of these simplices are not extrinsic orientations relating the simplices to external objects (e.g., fixed reference frames). Rather, the orientation of a simplex as used here is an intrinsic property of the relative distributions of the simplex vertices among themselves. For a planar triangle, its orientations (denoted by "+" and "-" here as in our earlier papers) indicate whether the vertices of the triangle form a counterclockwise or clockwise cycle when traversed in some specified order. The tetrahedron orientation signs are likewise

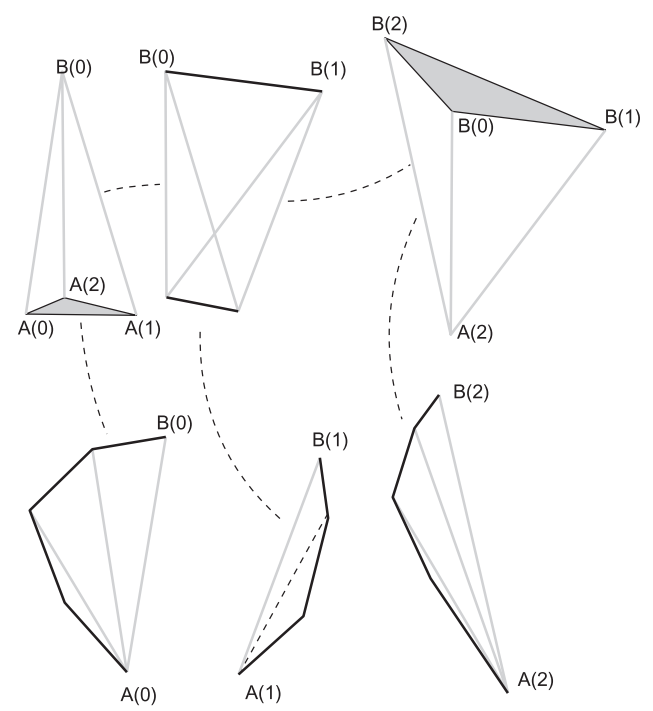

Fig. 3. Major part of a simplicial construction tree for the system in Fig. 1. defined. As an example, Fig. 4 shows two configurations and one tetrahedral tree of a triangular prism, i.e.a three-legged parallel platform with spherical joints at both ends of the legs. In the figure, the fixed links and diagonals are drawn in black and in gray respectively. The pictured configurations differ only in the opposite orientations of $\operatorname{Tet}(3)$. For more about orientation parameters, see [8], [12].

These simplex-based parameters can be used to parametrize the example hand system, either open or grasping, in a unified way. When the hand grasps an object with all three fingers at fixed contact points on the objects, we model the grasp by fixed link lengths of the diagonal among the tips. Then the system loses 3 DOF but can still be explicitly parametrized by the simplex-based parameters, by eliminating from the parameter set the now fixed lengths of the diagonals between the fingertips, $[A(0) A(1)],[A(0) A(2)],[A(1) A(2)]$. (When only two fingers grasp an object, the explicit parametrization set is obtained by eliminating just one diagonal length.)

Also note that the simplex assembly process as described earlier can be considered as an algorithm for the forward kinematics for determining the joint positions and thus system configurations from the simplex-based parameters. The inverse kinematics problem, when considered for some fixed fingertip positions or desired inter-fingertip distances (such as those arising from grasping an object at fixed contacts), is roughly equivalent to finding the valid parameter values of the system CSpace, in other words, the CSpace as parametrized by the simplex-based parameters, which is the main topic of this paper and will be discussed next.

\section{B. Simplex Formation Constraints}

In our new simplex-based parameters, the formulations of loop closure constraints are fundamentally different from the conventional ones for joint parameters or Cartesian coordinates. The key point is that

each loop can be formed if and only if all the simplices in its part of the construction tree can be successfully formed.

Therefore, we formulate the loop closure constraints as simplex formation constraints, such as triangle inequalities for triangles and Cayley-Menger determinant constraints [2] for general simplices in Euclidean space. Further, these constraints are in shape parameters only, since only the shape parameters, along with fixed link lengths, determine the existences and shapes of simplices. Orientation parameters are independent of the loop closure constraints.

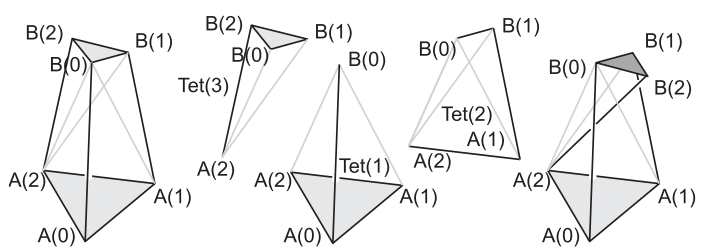

Fig. 4. A triangular prismoid: two configurations (at both ends) and a construction tree of 3 tetrahedra, with Tet(2) adjacent to the other two (because it has common triangular faces with the other two). The two configurations differ only in the orientation of Tet(3). 
Below we recite the well-known simplex formation constraints on the side lengths of triangles and tetrahedra in Euclidean space, which are the only simplices used in the example systems in this paper. Denote the vertices of a triangle by $P(i), i=1,2,3$. Their interpoint distances must satisfy the following triangle inequalities.

$$
\begin{aligned}
& d(1,2)-d(2,3)-d(3,1) \leq 0 \\
& d(2,3)-d(3,1)-d(1,2) \leq 0 \\
& d(3,1)-d(1,2)-d(2,3) \leq 0
\end{aligned}
$$

Equivalently, we can require non-negativity of the following Cayley-Menger determinant, which is proportional to the area of the triangle.

$$
2\left(\frac{-1}{2}\right)^{3}\left|\begin{array}{cccc}
0 & 1 & 1 & 1 \\
1 & 0 & D(1,2) & D(1,3) \\
1 & D(2,1) & 0 & D(2,3) \\
1 & D(3,1) & D(3,2) & 0
\end{array}\right| \geq 0
$$

(Here $D(i, k)=d^{2}(i, k)$ is the squared distance between ex points $P(i)$ and $P(k)$.) Similarly, for a tetrahedron with vertices $P(i), i=1, \ldots, 4$ and squared side lengths $D(i, j)$ $(0 \leq i, j \leq 3)$, existence is equivalent to the CMD constraint

$$
\left|\begin{array}{ccccc}
0 & 1 & 1 & 1 & 1 \\
1 & 0 & D(0,1) & D(0,2) & D(0,3) \\
1 & D(1,0) & 0 & D(1,2) & D(2,3) \\
1 & D(2,0) & D(2,1) & 0 & D(2,3) \\
1 & D(3,0) & D(3,1) & D(3,2) & 0
\end{array}\right| \geq 0 .
$$

In both (2) and (3), equality is equivalent to singularity of the corresponding simplex (collinearity of the vertices of a triangle, coplanarity of the vertices of a tetrahedron).

\section{An Example Simplicial Construction Tree}

For the simplices drawn in Fig. 3 for the three-fingered grasping system in Fig. 1, the main idea is to use the base-totip diagonals $[A(i) B(i)]$ and inter-tip diagonals $[A(i) A(i+1]$, together with the links $[B(i) B(i+1)]$ between the fixed bases, to form a virtual triangular prism. Then define three more diagonals, such as $[A(1) B(0)],[A(2) B(0)]$, and $[A(2) B(1)]$ (see Fig. 3) to form a construction tree of three tetrahedra for the prism, in a way similar to Fig. 4. (Clearly other choices of three diagonals could be made, resulting in different trees.)

Now for each finger, its base-to-tip diagonal forms a virtual loop with its links. Because all the internal joints are modeled to be revolute, with the rotational axes parallel to each other and perpendicular to the finger plane, this finger loop can be considered to be a loop with revolute joints contained in a plane (that rotates with the base spherical joint); and we have studied construction trees for planar loops with revolute joints in our previous papers, to which we refer the reader for further details ( [11], [12] deal only with the loop closure constraint, [13] extends our study to self-collision avoidance). Briefly, a planar loop with $n$ revolute joints has a triangle construction tree with $n-3$ diagonals and $n-2$ triangles (for $n>3$ this tree is not unique; in Fig. 3 we have used the construction tree

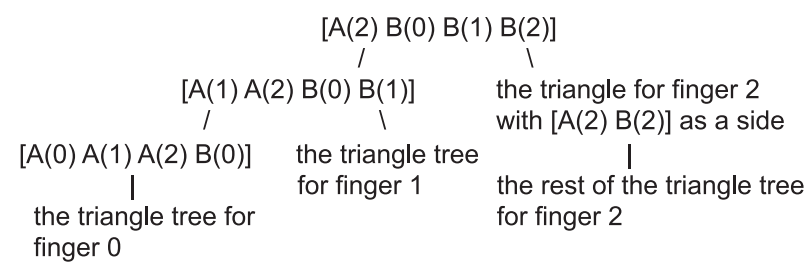

Fig. 5. A construction tree for the example 3-fingered manipulation system (the triangle trees for finger loops are not given in detail).

of triangles anchored at the tip joints, mostly for simplicity of the figures-other trees certainly could be used instead).

To keep the figure simple and use it to reflect main ideas in our approach, we do not reflect in Fig. 3 one subtlety existing in the simplicial construction for the hand system drawn in Fig. 1, namely, how to determine the finger planes. Note that each finger shares its base-to-tip diagonal with an appropriate tetrahedron and is adjacent to the tetrahedron in the construction tree. Suppose that in the simplex assembly process, we place the tetrahedra first. Then to put down the triangles for the finger loops, we need to be able to determine the orientations of the finger planes. These orientations will largely depend on the particular realizations (actuations) of the spherical joints. In our preliminary study, we have found that we can build upon the diagonals and the construction trees in Fig. 1 to accommodate various possible realizations of the spherical joints, sometimes by introducing additional diagonals. These new diagonals increase the codimension of the corresponding configuration spaces in their new ambient spaces; but their codimensions are still generally smaller than they would be in joint angles space. We will leave these issues to a future paper. Here we'd like to introduce an extra degree of freedom for each finger, which is to allow each finger plane to rotate freely about its end-to- tip diagonal.

Note that, with our human fingers, when we keep the base and tip of a finger fixed in space, we can still bend (rotate) the finger plane a little bit around the base-to-tip diagonal, probably allowed by the tendons in the hand. While the models of these behaviors and their engineering implications need more careful examination, here we will use this model to demonstrate the applicability of the simplex-based approach to highly complex manipulation type systems. We also note that such a model can still be uesful for applications in areas such as computer animation, where actuations and engineering feasibilities are not as critical as for physical systems.

For this extra degree of freedom for each finger, we use a dihedral angle between the plane of the finger with one triangular face of its adjacent tetrahedron. The overall system now has 17 degrees of freedom; its simplicial construction tree is indicated in Fig. 5. The simplex-based parameters are

- shape parameters (for the shapes of the simplices): lengths of the 14 diagonals, and

- orientation parameters (for the relative configurations of adjacent simplices): the 3 dihedral angles and 11 essentially binary orientations of the simplices. 


\section{Configuration Space Parametrizations}

The simplex tree shown schematically in Fig. 5 has 3 tetrahedra, and 8 triangles for the finger loops (not explicitly shown in the tree) of the hand system in Fig. 1. (As mentioned earlier, this system has other trees, but this one will do to explain our approach.) Each simplex imposes some inequality constraints on the shape parameters involved in it. Taken together, all these simplex formulation constraints define the set of feasible shape parameters, which we call DStretch. For this system, squared diagonal lengths can serve well as shape parameters, partly because both triangle and tetrahedron constraints can be formulated in them as the CayleyMenger determinant constraints (2)(3). But it is also fine to use a combination of squared diagonal lengths (for those involved in tetrahedra) and direct diagonal lengths(for those only involved in triangles). More generally, it is possible to use non-degenerate (diffeomorphic) functions to map the diagonallength-based shape parameters to other parameters.

Of the 14 diagonals listed at the beginning of section III, some will have fixed lengths for some grasp configurations. For example, when the hand grasps an object with all three fingers at fixed contact points on the objects, the three diagonals $[A(0) A(1)],[A(0) A(2)],[A(1) A(2)]$ between the fingertips will have fixed lengths and should be eliminated from the shape parameter set for the system configurations in this grasp mode. Similarly, for all configurations with two fingers grasping an object at fixed contacts, the diagonal between the corresponding two fingers has a fixed length and is no longer needed for the shape parameterization. Similarly, if we are interested in fixing the base-to-end distance of some finger, we can use that distance for the relevant diagonal and exclude the diagonal from the shape parameter set.

In summary, the set of 14 diagonals and its appropriate subsets provide explicit parametrizations of the shapes of simplices in the construction tree. These shape parameters are subject to simplex formation constraints, as well as fixed length constraints for suitable formulation of grasping modes.

As mentioned above, the loop closure constraints impose no constraints on the set of feasible orientation parameters, which we call DFlip. For the hand system and its simplicial tree in Fig. 5, DFlip $=\left(S^{1}\right)^{3} \times\{+,-\}^{11}$, where the threedimensional torus $\left(S^{1}\right)^{3}$ reckons dihedral angles and $\{+,-\}^{11}$ intrinsic simplex orientations. Note that if we want to fix the value of one dihedral angle or the orientation sign of one simplex, we can take out its corresponding orientation parameter from the set of system orienation parameters. Also note that the joint limits existing in human hands (for most of us) generally allow each finger to bend only toward the palm, with its fully extended configuration as one extreme feasible configuration. If we impose similar joint angle constraints on a robotic hands, and use anchored triangles in the construction tree (as in our example), then we need consider just one orientation for each triangle, so that the triangle-related orientation signs drop out of DFlip. As will become clear soon, a smaller set of orientation parameters will substantially simplify the overall structure of CSpace (by reducing the number of copies of DStretch in CSpace).

Singular configurations play an important role in constructing the overall configuration spaces. Their shape and orientation parametrization need careful treatments. Refer to papers [9], [11], [12] for our approaches to similar issues for loops with spherical type joints. Here, by labeling singular simplices-like a tetrahedron (resp., triangle) with 4 (resp., 3) coplanar (resp., collinear) vertices - with both + and - , we obtain (roughly) an identification of the configuration space with DStretch $[\mathcal{T}] \times$ DFlip. In other words, using the simplicial construction tree in Fig. 5, the configuration space of the hand system in Fig. 1 consists of $2^{11}$ copies of DStretch $\times\left(S^{1}\right)^{3}$. The overall parametrization and stratified structures of the CSpace here is very similar to that for a planar loop with $n$ revolute joints described in [11], [12], but with some important technical differences, such as more complicated constraints on DStretch and more complex gluing of different strata. Further, the overall structures of the configuration spaces will depend on the identification (gluing) of different strata along proper singular subsets; and the identification of the hand system will be more complicated than that of a planar polygon. We will address these topics in future papers. Here we summarize the main parametrization results whose proofs we have sketched in this section.

Theorem 1. Consider the 3 -fingered hand system in Fig. 1 and its simplicial construction tree in Fig. 5. Then:

(A) The configurations of the system are described by simplex-based parameters.

(B) The system configuration space CSpace is essentially the product of DStretch and DFlip, where (1) DStretch comprises shape parameters satisfying explicit, simply evaluated constraints (triangle or Cayley-Menger determinant inequalities, and range inequalities) required for successful simplex formation, and is a convex body in squared diagonal lengths, while (2) DFlip comprises relative orientation parameters, and is independent of loop closure constraints.

\section{EXAmple System II: Star MAnipulator}

Our second example system is the general star-manipulator, as defined in [17], [25]. Such a manipulator is formed by joining $k$ planar chains with revolute joints to a common point (like the thorax of an insect) and then fixing the base of each chain to the ground (an example is shown, in heavy black lines, in the leftmost subfigure of Fig. 6). The result is a planar linkage system with multiple loops (for $k \geq 3$ ). The designers of these manipulators call the chains "legs". We will call them "fingers" for the sole purpose of using the same hand terminology for the example systems in this paper.

We assume that each finger has at least 2 joints so as to give a nontrivial reachable workspace for the thorax $A$. Consider the thorax $A$ to be the tip of all the fingers. Its position is determined by the finger link lengths and joint angles. For a manipulator with $k$ fingers, the condition that all $k$ fingertips meet at one common point in the plane imposes $2(k-1)$ constraints on the joint angles. So if we use joint angles as 
the parameters for the study of a star-shaped manipulator with $k$ fingers, the configuration space is an semi-analytic set of codimension $2(k-1)$ in the ambient joint angle space.

Alternatively, if we know the position of the thorax $A$, we can define an auxiliary link between $A$ and each base $B(i)$ (drawn as dashed lines in Fig. 6). Each such auxiliary link forms a (virtual) loop with the original links of the corresponding finger, and the loop is a planar loop with revolute joints, whose configurations can be studied via its construction trees of triangles (for instance, anchored triangles as shown in Fig. 3). See [8], [11]-[13] for more details.

The position of $A$ is parametrized by its Cartesian coordinates $\left(A_{x}, A_{y}\right)$, and its range (the workspace of $A$ ) is easily found. Specifically, the reachable workspace of the fingertip of the open chain with revolute joints and fixed base $B(i)$ is generally an annulus (exceptionally, a disk) with center at $B(i)$, having inner and outer radii that are easily computed from the finger link lengths. Such an annulus workspace corresponds to the minimum and maximum bounds on the distance between the finger tip and the base.

$$
(\text { min-radius- } i)^{2} \leq i D(A, B(i)) \leq(\text { max-radius }-i)^{2}
$$

When the fingers are assembled to form the star-manipulator, the workspace of $A$ is the intersection of these annuli given by inequalities (4), $i=0, \ldots, k-1$. The explicitness (and comparative simplicity) of this workspace, along with some critical insights and novel ideas, was used in [17], [25] to develop a smart polynomial time algorithm for a complete path planner (ignoring all constraints other than loop closure) for these manipulators; the algorithm uses Cartesian coordinates on the workspace, and joint angle parameters for the figners.

Here we focus on parametrization rather than motion planning, using generalized simplex-based parameters for a starmanipulator, as follows:

- Cartesian coordinates for the thorax $A$ : $\left(A_{x}, A_{y}\right)$;

- shape parameters for the triangles in the construction trees of the finger loops: lengths of the diagonals; and

- orientation parameters for the triangles in the construction trees of the finger loops: essentially binary variables for the intrinsic triangle orientations.

In these parameters, the system constraints are the radius constraints (4) (on $\left(A_{x}, A_{y}\right)$ ), and triangle formation constraints (parametrized by $\left(A_{x}, A_{y}\right)$ ) on triangle shape parameters. There are no constraints on the orientation parameters.

Our general methods can be used to show that this parametrization has the following properties.

Theorem 2 Consider a star-shaped manipulator and its generalized simplex-based parameters. Then:

(A) The configurations of the system are described by the generalized simplex-based parameters.

(B) The system configuration space CSpace essentially the product of DStretch and DFlip, where (1) DStretch, the set of feasible length parameters, comprises the Cartesian coordinates of the thorax and triangle shape parameters satisfying explicit, simply evaluated constraints (the radii, and triangle or
Cayley-Menger determinant inequalities) required for reachable thorax positions and successful simplex formation, while (2) DFlip comprises triangle orientation parameters, and is independent of loop closure constraints.

Again CSpace, as parametrized by the generalized simplexbased parameters, consists of copies of DStretch. Fig. 6 shows a schematic figure of DStretch for a 3-DOF star-manipulator. The 3-jointed finger has one diagonal, which has an interval of feasible values for each thorax position; the 4 jointed finger has 2 diagonals, with a convex polygonal region of feasible values. So DStretch is a family of 3-dimensional prisms parametrized by the points of the reachable workspace $W S(A)$ of $A$.

The similarity in the parametrization and structures of the configuration space of these two example systems are common for linkage systems allowing simplicial construction trees. The example systems and their simplicial construction trees presented here are substantially different from those in our earlier papers [8]-[13], and the encouraging results to these challenging multi-loop systems here further demonstrate the versatility of the simplex-based approach.

Many important linkage systems (e.g., general $6 R$ manipulators and many multivertex origamis) can be shown not to allow simplicial construction trees. Nonetheless, using methods related to simplicial construction trees, we have derived some interesting results for several such systems; for example, we can recover the number of inverse kinematics solutions for $6 R$ manipulators in a natural way. Here we have no space for further details, which will appear in later papers.

\section{SUMMARY}

Linkage systems such as multi-fingered hands and multilegged mobile systems are important in the study of robotics. They are also very challenging - in part because that the limbs form loops when their tips contact some common objects such as an grasped object for a hand or a common ground for legs. Conventionally the configurations of these systems are described in joint parameters, and the corresponding configuration spaces are lower-dimensional semi-algebraic subsets (as defined by loop closure constraints) embedded in joint parameter space. Such an implicit parametrization, coupled with complex nonlinear formulation of the loop closure constraints, poses challenges for diverse problems such as computing CSpace structures and using sampling for motion planning.

In contrast, explicit parametrization, where the cardinality of the set of parameters is equal to the dimension of the configuration space under study, offers advantages over implicit parametrization, especially when the constraints on the explicit parameters have nice properties. In this paper, we present explicit parametrization for two example anthropomorphic manipulation systems. Our parametrization are based on our recently developed simplicial construction trees for multi-object systems; and the constraints on the simplexbased parameters are mainly simplex formation constraints (such as triangle inequalities and Cayley-Menger determinant constraints). The model systems here are difficult to deal with using the joint angle parameters; and our results here, along 

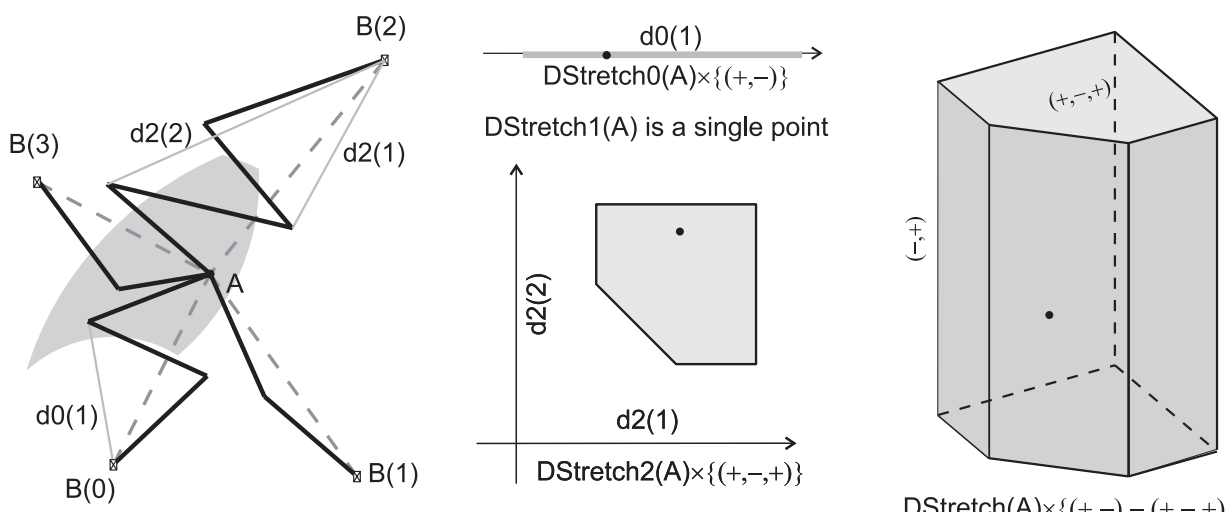

DStretch3(A) is a single point

$\operatorname{DStretch}(\mathrm{A}) \times\{(+,-),-,(+,-,+),+\}$

Fig. 6. Schematic view of DStretch of a star-manipulator.

with those in our earlier papers [8]-[13], demonstrate the generality and effectiveness of the simplex-based approach.

Not every linkage system has a simplicial construction tree. But in our preliminary study of other linkages, joint types and system constraints, we have found that many kinematic structures can accommodate triangle or tetrahedral decompositions, sometimes by first ignoring some constraints in the systems. Such structures often lead to parametrizations of configuration spaces with lower codimensions than those parametrized by joint parameters. In ongoing research, we are generalizing our simplex-based approach, and developing algorithms to identify and create systems with simplicial construction trees.

\section{ACKNOWLEDGMENT}

We are partially supported by NSF grant IIS0713335. We thank the anonymous referees for their helpful remarks.

\section{REFERENCES}

[1] M. Alberich, F. Thomas, and C. Torras, Flagged parallel manipulators, IEEE Trans. Robotics (2007), 1013-1023.

[2] L. M. Blumenthal, Theory and applications of distance geometry, 2nd edition, American Mathematical Society, Providence, 1970.

[3] H. Choset, W. Burgard, S. Hutchinson, G. Kantor, L. E. Kavraki, K. Lynch, and S. Thrun, Principles of robot motion: Theory, algorithms, and implementation, MIT Press, Cambridge, MA, 2005.

[4] John J. Craig, Introduction to robotics: Mechanics and control, 2nd edition, Addison-Wesley Publishing Company, Reading, MA, 1989.

[5] M. Cutkosky, Grasping and fine manipulation for automated manufacturing, Kluwer Academic Publishers, Boston, 1986.

[6] B. Goodwine and J. Burdick, Motion planning for kinematic stratified systems with application to quasi-static legged locomotion and finger gaiting, Algorithmic and Computational Robotics - New Directions (WAFR 2000), 2000, pp. 109-127.

[7] R. Grupen and O. Brock (signatories R. Ambrose, C. Atkeson, O. Brock, R. Brooks, C. Brown, J. Burdick, M. Cutkosky, A. Fagg, R. Grupen, J. Hoffman, R. Howe, M. Huber, O. Khatib, P. Khosla, V. Kumar, L. Leifer, M. Mataric, R. Nelson, A. Peters, K. Salisbury, S. Sastry, and R. Savely), White paper: Integrating manual dexterity with mobility for human-scale service robotics - the case for concentrated research into science and technology supporting next-generation robotic assistants, http: // robotics. cs.umass . edu/ oli/publications/index.html, 2004.

[8] L. Han and L. Rudolph, The inverse kinematics of a serial chain with joints under distance constraints, Robotics: Science and Systems, 2006.

[9] _ A unified geometric approach for the inverse kinematics of a serial chain with spherical joints, Proc. IEEE Int. Conf. Robot. Autom. (ICRA), 2007.
[10] Simplex-tree based kinematics of foldable objects as multi-body systems involving loops, Robotics: Science and Systems, 2008.

[11] L. Han, L. Rudolph, J. Blumenthal, and I. Valodzin, Stratified deformation space and path planning for a planar closed chain with revolute joints, Proc. Seventh International Workshop on Algorithmic Foundation of Robotics (WAFR), 2006.

[12] _ Convexly stratified deformation space and efficient path planning for a planar closed chain with revolute joints, Int. J. Robot. Res. 27 (2008), 1189-1212.

[13] L. Han, L. Rudolph, S. Dorsey-Gordon, D. Glotzer, D. Menard, J. Moran, and J. R. Wilson, Bending and kissing: Computing self-contact configurations of planar loops with revolute joints, Proc. IEEE Int. Conf. Robot. Autom. (ICRA), 2009.

[14] M. Kapovich and J. Millson, On the moduli spaces of polygons in the euclidean plane, J. Diff. Geom. 42 (1995), 133-164.

[15] J. C. Latombe, Robot motion planning, Kluwer Academic Publishers, Boston, 1991.

[16] S. M. LaValle, Planning algorithms, Cambridge University Press, Cambridge, U.K., 2006, http://planning.cs.uiuc.edu/.

[17] G.F. Liu, J.C. Trinkle, and N. Shvalb, Motion planning for a class of planar closed-chain manipulators, Proc. IEEE Int. Conf. Robot. Autom (ICRA), 2006, pp. 133-138.

[18] M. Mason, Mechanics of robotic manipulation, MIT Press, Cambridge, 2001.

[19] M. Mason and K. Salisbury, Robot hands and the mechanics of manipulation, MIT Press, Cambridge, 1985.

[20] J.-P. Merlet, Parallel robots, Springer, New York, 2000.

[21] R.J. Milgram and J.C. Trinkle, The geometry of configuration spaces for closed chains in two and three dimensions, Homology Homotopy and Applications (2002).

[22] R. M. Murray, Z. Li, and S. S. Sastry, A mathematical introduction to robotic manipulation, CRC Press, Boca Raton, 1994.

[23] J. Ponce, S. Sullivan, A. Sudsang, J.-D. Boissonat, and J.-P. Merlet, On computing four-finger equilibrium and force-closure grasps of polyhedral objects, Int. J. Robot. Res. 16 (1996), 11-35.

[24] J. Porta, L. Ros, and F. Thomas, On the trilaterable six-degree-offreedom parallel and serial manipulators, Proc. IEEE Int. Conf. Robot. Autom. (ICRA), 2005.

[25] N. Shvalb, L.G. Liu, M. Shoham, and J.C. Trinkle, Motion planning for a class of planar closed-chain manipulators, Int. J. Robot. Res. 26 (2007), no. 5, 457-474.

[26] X. Tang, S. Thomas, and N. Amato, Planning with reachable distances, Proc. Eighth International Workshop on Algorithmic Foundation of Robotics (WAFR), 2008.

[27] C. Torras, F. Thomas, and M. Alberich, Stratifying the singularity loci of a class of parallel manipulators, IEEE Trans. Robotics (2006), 23-32.

[28] J.C. Trinkle and R.J. Milgram, Complete path planning for closed kinematic chains with spherical joints, Int. J. Robot. Res. 21 (2002), no. 9, 773-789.

[29] Y. Wei and B. Goodwine, Stratified manipulation on non-smooth domains, IEEE Trans. Robotics and Automation 20 (Feb. 2004), no. 1, 128-132. 